\title{
Técnicas de aislamiento y concentración de volátiles de aceites vegetales
}

\author{
Por M. T. Morales, R. Aparicio y F. Gutiérrez \\ Instituto de la Grasa y sus Derivados \\ Avda. Padre García Tejero, 4. 41012-Sevilla
}

\section{RESUMEN}

Técnicas de aislamiento y concentración de volátiles de aceites vegetales

Uno de los métodos de evaluación del flavor de los alimentos consiste en la determinación de sus componentes volátiles. La primera etapa necesaria para realizar este tipo de análisis es el aislamiento y concentración de los mismos. Se ha realizado una revisión bibliográfica sobre las diferentes técnicas utilizadas para este fin en diferentes alimentos y especialmente en aceites vegetales. Los métodos utilizados son muy diversos, se ofrece una explicación de cada uno de ellos considerando las diferentes variantes existentes. Asimismo se detallan las aplicaciones realizadas en aceites vegetales durante los últimos años.

PALABRAS-CLAVE: Aceite vegetal - Componentes volátiles (aislamiento) - Componentes volátiles (concentración) - Información (articulo).

\section{SUMMARY}

Techniques for the isolation and concentration of vegetable oils volatiles

One of the methods for food flavor evaluation is the analysis of volatile components. First step in this kind of analysis is the isolation and concentration of volatiles. A review is carried out about different techniques applied in foods and especially in vegetable oils. Very different methods have been used, an explication of each one with its different cases are presented. Likewise the applications carried out in vegetable oils during last years are discussed.

KEY-WORDS: Information (paper) - Vegetable oil - Volatile components (concentration) - Volatile components (isolation).

\section{INTRODUCCION}

Los volátiles de los alimentos juegan un papel primordial y fundamental en el flavor siendo éste una sensación muy compleja compuesta primariamente de olor y gusto, complementada también por sensaciones táctiles y quinestésicas.

Antes de abordar el estudio de la fracción aromática de los alimentos hay que tener en cuenta los siguientes puntos (Flath, 1981): a) la concentración de volátiles en la muestra suele ser baja por lo que se va a obtener una fracción pequeña de los mismos a partir de una cantidad de muestra grande; b) la fracción volátil está constituida generalmente por un gran número de componentes de peso molecular y naturaleza química diferentes y cuyas concentraciones pueden variar en un intervalo muy amplio; c) no hay una correspondencia directa sobre la incidencia de cada compuesto en el aroma de un alimento y su concentración en la fracción aromática ya que los umbrales de detección son muy variables; d) en la etapa de extracción al utilizar disolventes se hace necesario un proceso de concentración y e) hay que evitar la formación de artefactos durante el análisis ya que pueden interferir y dar lugar a resultados erróneos.

En general (Mehlitz, 1962), el análisis de la fracción volátil de los alimentos requiere cuatro etapas perfectamente definidas: 1) separación de la fracción volátil de la muestra; 2) concentración de la misma; 3) fraccionamiento en los diferentes componentes y 4) identificación.

Todas estas etapas son de gran importancia en este tipo de análisis y deben ser cuidadosamente elegidas (Golovnya, 1982). En el presente trabajo se ha llevado a cabo una puesta al día sobre los diferentes métodos utilizados para realizar las dos primeras tanto en aceites vegetales como en otro tipo de alimentos. Estos métodos podemos agruparlos en dos grandes grupos:

A) Aquellos que no realizan concentración de la muestra.

- Inyección directa.

- Espacio de cabeza estático.

B) Aquellos que realizan concentración.

- Destilación.

- Extracción.

- Espacio de cabeza dinámico.

- Arrastre y atrape.

Estos métodos son los más utilizados aunque también se han aplicado otros tales como la extracción con disolventes y diálisis por membrana que ha sido aplicada a muestras de queso Cheddar y carne de buey (Benkler, 1980); la técnica de difusión simple, aplicable especialmente a líquidos viscosos y espumosos (Booker, 1985) o la extracción con fluido supercrítico, menos utilizada hasta la fecha, pero que ha sido aplicada con éxito a especias, chicle y corteza de naranja (Hawthorne, 1988). 


\section{METODOS QUE NO REALIZAN CONCENTRACION}

\subsection{Inyección directa}

Esta es la técnica más simple para el análisis de sustancias volátiles y la que requiere menor manipulación de la muestra, aunque tiene el inconveniente de ser la menos sensible (Mordret, 1985), además es necesario utilizar temperaturas elevadas, por lo que en los volátiles analizados también aparecen productos de descomposición. No es de aplicación generalizada en los alimentos, sin embargo, se observa en la bibliografía una elevada utilización de este método en aceites vegetales, principalmente en estudios de oxidación (Morrison, 1981), (Warner, 1985).

En esencia el método consiste en colocar una pequeña cantidad de muestra sobre un soporte (generalmente lana de vidrio) a la entrada del inyector o en una precolumna adosada a él, posteriormente la muestra se calienta y se purga con el gas portador, pasando los volátiles a la columna cromatográfica.

Se han desarrollado una amplia gama de variantes sobre este método, así el método puesto a punto por Dupuy (1971) consiste en la colocación de una pequeña cantidad de muestra en un tubo relleno de lana de vidrio colocado a la entrada del inyector, la muestra se purga con gas y se calienta a una temperatura determinada, de manera que se produce una extracción de los volátiles que son arrastrados por el gas portador hasta el interior de la columna para ser cromatografiados. Este método con algunas modificaciones (Dupuy, 1973) se ha aplicado a la determinación de sustancias volátiles en aceites refinados y shortenings relacionando su contenido con la calidad, comprobándose que los aceites de mejor calidad presentaban menor contenido en volátiles. También se ha utilizado una adaptación de esta técnica (Warner, 1985) que consiste en colocar $15 \mu$ lde muestra en un tapón de lana de vidrio en la entrada del inyector para determinar la estabilidad del flavor del aceite de soja midiendo los períodos de inducción basados en el tiempo requerido para la formación rápida de sustancias volátiles bajo condiciones de almacenamiento conocidas; esta misma adaptación del método de Dupuy se utilizó para, aplicándolo a aceites crudos, predecir la estabilidad del flavor de aceites refinados durante su almacenamiento (Warner, 1988). La misma técnica fue también empleada para el estudio de volátiles de aceites oxidados en diferentes condiciones, comparándola con otras dos técnicas de obtención de volátiles (Snyder, 1988); asimismo se ha aplicado al estudio de los efectos de antioxidantes, envases y luz en la calidad del aceite de girasol (Morrison, 1981), estableciendo correlaciones entre contenido de volátiles y análisis sensorial.

El método de Dupuy fue posteriormente mejorado de manera que se utiliza un dispositivo de entrada especial para poder aumentar el tamaño inicial de muestra sin concentrarla (Dupuy, 1985). También se recoge en la bibliografía un sistema automatizado de inyección directa que introduce el tubo relleno de lana de vidrio con la muestra en un "bloque de entrada externo", conectado al cromatógrafo, en el cual se produce el aislamiento de volátiles que son después purgados a la columna (Gensic, 1984), el método es aplicado a aceites durante su procesado, obteniéndose rápidos y reproducibles perfiles de volátiles.

Por otra parte Jackson y Giacherio (1977) desarrollaron un procedimiento directo, consistente en colocar la muestra de aceite sobre la lana de vidrio que se encuentra rellenando un extremo de un tubo de aluminio en forma de "U", calentarlo posteriormente y purgarlo con gas, de manera que los volátiles son barridos a una columna cromatográfica que está conectada al otro extremo del tubo, después se desconecta la columna del tubo en " $U$ " y se conecta al cromatógrafo para continuar el análisis. Una modificación de este procedimiento (Jackson, 1981) que consiste en utilizar un tubo más largo se ha aplicado a la determinación de volátiles en concentrados de soja. Min (1981) utilizó una nueva variación en la cual el tubo en "U" se rellena con lana de vidrio, sulfato sódico anhidro, lana de vidrio, carbonato potásico y nuevamente lana de vidrio, los dos nuevos componentes añadidos sirven para eliminar el agua y los compuestos ácidos de la muestra; la técnica se utilizó para estudiar la evaluación del flavor y establecer correlaciones entre el análisis sensorial e instrumental; para el aislamiento de volátiles en el estudio de la estabilidad oxidativa de aceites de soja durante el almacenamiento (Min, 1983a) y también para correlacionar el análisis sensorial realizado por paneles de 8 laboratorios diferentes con el análisis instrumental por cromatografía gaseosa en aceites de soja y maíz (Min, 1983b).

\section{Tabla I}

Parámetros y referencias de los métodos por Inyección Directa

\begin{tabular}{|c|c|c|c|c|c|}
\hline \multirow{2}{*}{$\begin{array}{l}\text { Cantidad de } \\
\text { muestra } \\
1 \mathrm{ml} \\
\text { (soja, maíz) }\end{array}$} & \multicolumn{2}{|c|}{$\begin{array}{c}\text { Inyección } \\
T^{2}\left({ }^{\circ}\right) t(\text { min })\end{array}$} & \multirow{2}{*}{$\frac{\begin{array}{c}\text { Gas } \\
\text { portador }\end{array}}{\mathrm{He}}$} & \multirow{2}{*}{$\begin{array}{l}\text { Columna } \\
10 \% \text { polyMPE } \\
\text { Tenax } 80 / 100\end{array}$} & \multirow{2}{*}{ 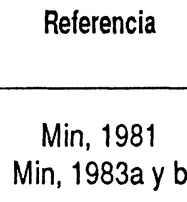 } \\
\hline & 160 & 15 & & & \\
\hline $\begin{array}{l}6-10 \text { gotas } \\
\text { (girasol) }\end{array}$ & 170 & 20 & - & $\begin{array}{l}7 \% \text { polyMPE } \\
\text { Tenax } 60 / 80\end{array}$ & Morrison, 1981 \\
\hline $\begin{array}{l}500 \mathrm{mg} \\
\text { (soja) }\end{array}$ & 220 & 25 & $\mathrm{~N}_{2}$ & $\begin{array}{l}8 \% \text { polyMPE } \\
\text { Tenax } 60 / 80\end{array}$ & Gensic, 1984 \\
\hline $\begin{array}{l}15 \mu \mathrm{l} \\
\text { (soja) }\end{array}$ & 180 & - & $\mathrm{He}$ & $\begin{array}{l}8 \% \text { polyMPE } \\
\text { Tenax } 60 / 80\end{array}$ & Warner, 1985 \\
\hline $\begin{array}{l}300 \mathrm{mg} \\
\text { (soja) }\end{array}$ & 200 & - & $\mathrm{N}_{2}$ & SE - 54 & Dupuy, 1985 \\
\hline $\begin{array}{l}15 \mu \\
\text { (soja) }\end{array}$ & 180 & - & $\mathrm{He}$ & Durabond-5 & Warner, 1988 \\
\hline $\begin{array}{l}2 \mu l \\
\text { (soja) }\end{array}$ & 180 & 5 & $\mathrm{He}$ & Durabond-5 & Snyder, 1988 \\
\hline $\begin{array}{l}0,6-0,7 \mu \mathrm{l} \\
\text { (oliva virgen) }\end{array}$ & 160 & - & $\mathrm{H}_{2}$ & CP Sil 13 & Mariani, 1990 \\
\hline
\end{tabular}


Otro método de inyección directa utilizado lleva una precolumna con un dispositivo para retener el aceite inyectado (NGD C78, procedimiento $B$ ), se usa en aceite de oliva virgen en el análisis de compuestos halogenados volátiles, estableciéndose las condiciones operativas adecuadas para la determinación de estos productos en presencia de tricloroetileno y tetracloroetileno, cosa que no es posible siguiendo la metódica oficial (Mariani, 1990).

Las condiciones cromatográficas de los distintos trabajos recientes que utilizan la técnica de inyección directa se encuentran recogidas en la Tabla I. Se puede observar que la cantidad de muestra que se utiliza es pequeña por lo que es una técnica poco sensible, siendo además necesario el uso de temperaturas elevadas, por lo cual pueden aparecer productos de degradación, y teniendo el inconveniente de que hay que hacer una buena limpieza entre cada muestra para evitar el riesgo de aparición de "efectos memoria" en el cromatógrafo.

\subsection{Espacio de cabeza estático}

Es una forma sencilla de analizar la fracción volátil de un alimento. Consiste en el análisis de una alícuota de la fase de vapor que se halla en equilibrio con la muestra en un vial sellado y que se ha sometido durante un tiempo a una temperatura determinada. Este método requiere un control rígido de la temperatura y de la recogida de la muestra. Las concentraciones de los volátiles en ambas fases no cambian con el tiempo una vez alcanzado el equilibrio, sin embargo, sí pueden disturbarse temporalmente cuando se recoge la muestra por lo que hay que estudiar el método de recogida de la alícuota gaseosa con cuidado (Drozd, 1979) (Núñez, 1984).

Esta técnica tiene la gran desventaja de carecer de sensibilidad, esto unido a que con frecuencia suele haber grandes cantidades de agua en el espacio de cabeza de las muestras, la hace por tanto inadecuada para el análisis de trazas, donde se necesita realizar un paso previo de enriquecimiento, así como para el análisis de compuestos que tengan baja presión de vapor (Alberola, 1979a).

La técnica más usada de inyección de muestra es mediante jeringa, debido a una serie de ventajas (Mateos, 1990): no se introducen sustancias extrañas, se reducen al mínimo los cambios debidos a reacciones químicas y no hay pérdidas de las sustancias más volátiles. A pesar de todo posee limitaciones. Solamente es adecuada para los compuestos de alta volatilidad, es decir, para aquellos que están presentes en el espacio de cabeza en cantidades apreciables analíticamente (Jennings, 1977), la goma del tapón puede absorber determinados compuestos volátiles (Davis, 1970) y en el momento de llenar la jeringa se pueden producir fugas (Paillard, 1970).

El método se ha aplicado al análisis de volátiles de muestras tales como hierbas aromáticas (Chialva, 1983), cacahuetes (Sanders, 1989), bebidas (Rubico, 1988), salmuera (Montaño, 1990), etc. Sin embargo, en la bibliografía de los últimos años se encuentra una mayor aplicación del método a aceites.

En aceite de oliva virgen ha sido aplicada al estudio de las características aromáticas de aceites de distintas variedades y procedencias (Gutiérrez, 1975) y al estudio del problema del atrojado (Del Barrio, 1981 y 1983). También se ha utilizado en la evaluación de los defectos avinado, rancio y atrojado en este tipo de aceite (Gasparoli, 1986).

Asimismo ha sido utilizada como técnica comparativa en un estudio sobre predicción de la estabilidad del flavor de aceites de soja (Warner, 1985), y también en un estudio realizado con ocho aceites vegetales diferentes, frescos y con distintos niveles de oxidación, comprobándose que los volátiles identificados en cada caso provenian de la autoxidación de los ácidos grasos insaturados presentes en cada aceite (Snyder, 1985).

La técnica de espacio de cabeza estático también se ha aplicado al estudio de las relaciones entre contenido de volátiles y de ácidos grasos en aceites vegetales termoxidados, obteniéndose importantes diferencias entre aceites originales no calentados, pero no existiendo diferencias significativas entre aceites calentados con composición similar en ácidos grasos insaturados (Dobarganes, 1986). Esta técnica junto con las de espacio de cabeza dinámico e inyección directa se aplicó a aceites de soja en un estudio comparativo de métodos (Snyder, 1988) y también al estudio de volátiles halogenados en aceite de oliva virgen (Mariani, 1990).

Tabla II

\section{Parámetros y referencias de los métodos de espacio de cabeza estático}

\begin{tabular}{|c|c|c|c|c|c|}
\hline $\begin{array}{l}\text { Cantidad de } \\
\text { muestra }\end{array}$ & $\begin{array}{c}\text { SHS } \\
\Gamma(\mathcal{C}) 1 \text { (min) }\end{array}$ & $\begin{array}{l}\text { Inyección } \\
1 \text { (seg) }\end{array}$ & $\begin{array}{l}\text { Gas } \\
\text { portadox }\end{array}$ & Columna & Relerencia \\
\hline $\begin{array}{l}2 \mathrm{~g} \\
\text { (oliva virgen) }\end{array}$ & $70 \quad 30$ & 10 & $\mathrm{~N}_{2}$ & $\begin{array}{l}30 \% \text { Carbowax 20M } \\
\text { Chromosorb } 80 / 100\end{array}$ & $\begin{array}{l}\text { Del Barrio, } 1981 \\
\text { Del Barrio, } 1983\end{array}$ \\
\hline $\begin{array}{l}1 \mathrm{~g} \\
\text { (soja) }\end{array}$ & 18016 & - & $\mathrm{He}$ & $\begin{array}{l}\text { 8\% polyMPE } \\
\text { Tenax 60/80 }\end{array}$ & Warner, 1985 \\
\hline $\begin{array}{l}0,5 \mathrm{~g} \\
\text { (canola, maíz, } \\
\text { oliva, etc.) }\end{array}$ & 18010 & 30 & $\mathrm{~N}_{2}$ & Durabond-5 & Snyder, 1985 \\
\hline
\end{tabular}

\begin{tabular}{|c|c|c|c|c|c|c|}
\hline $\begin{array}{l}2 \mathrm{ml} \\
\text { (oliva virgen) }\end{array}$ & 80 & 30 & - & $\mathrm{N}_{2}$ & $\begin{array}{l}15 \% \text { Carbowax 20M } \\
\text { Chromosorb 80/100 }\end{array}$ & Gasparoli, 1986 \\
\hline $\begin{array}{l}1 \mathrm{~g} \\
\text { (palma, oliva, } \\
\text { girasol, soja) }\end{array}$ & 160 & 30 & 5 & $\mathrm{~N}_{2}$ & \multicolumn{2}{|c|}{$\begin{array}{l}30 \% \text { Carbowax 20M Dobarganes, } 1986 \\
\text { Chromosorb } 80 / 100\end{array}$} \\
\hline $\begin{array}{l}0,5 \mathrm{~g} \\
\text { (soja) }\end{array}$ & 180 & 20 & 60 & $\mathrm{He}$ & Durabond-5 & Snyder, 1988 \\
\hline $\begin{array}{l}3 \mathrm{~g} \\
\text { (oliva virgen) }\end{array}$ & 70 & 60 & - & $\mathrm{H}_{2}$ & CP Sil 13 & Mariani, 1990 \\
\hline $\begin{array}{l}0,5 \mathrm{~g} \\
\text { (colza, maíz, } \\
\text { girasol, etc.) }\end{array}$ & 90 & 30 & 30 & $\mathrm{He}$ & DB-1701 & Snyder, 1990 \\
\hline
\end{tabular}


Asimismo se recoge en la bibliografía el método de espacio de cabeza con extracción múltiple, la única diferencia con el simple consiste en inyectar una alícuota del espacio de cabeza del mismo vial varias veces, esperando que se restablezca el equilibrio en la muestra antes de cada inyección. Esta técnica ha sido aplicada a aceites vegetales de distinto tipo, realizándose tres inyecciones en cada caso y haciendo una comparación con el espacio de cabeza estático simple, observándose que se obtienen medidas más reproducibles utilizando este método (Snyder, 1990).

Las condiciones de cada uno de los trabajos anteriormente citados se recogen en la Tabla II, observándose que se utiliza una pequeña cantidad de muestra y que en casi todos los casos es necesario el empleo de temperaturas elevadas, ambas cosas hacen que esta técnica presente limitaciones, aunque tiene la ventaja de ser rápida y no requerir prácticamente manipulación de la muestra.

\section{METODOS QUE REALIZAN CONCENTRACION}

\subsection{Destilación-Extracción}

\section{Destilación}

Es una de las técnicas más utilizadas en el aislamiento de sustancias volátiles de los alimentos aunque hay que tener en cuenta que mediante ella pueden separarse también otros compuestos volátiles que no tienen repercusión en el aroma (Alberola, 1979b).

Los métodos más utilizados son la destilación a vacio y en corriente de vapor, siendo de gran interés la destilación a presión reducida que permite reducir la temperatura de trabajo, con lo cual se minimizan las posibles alteraciones de la muestra. Generalmente los vapores procedentes de la destilación se condensan en un refrigerante o bien son atrapados en diferentes trampas criogénicas o de materiales absorbentes, aunque esto último es menos frecuente.

Una vez finalizada la destilación puede inyectarse directamente el destilado en el cromatógrafo, pero suele ser necesaria una etapa de concentración ya que algunos compuestos pueden encontrarse a niveles de trazas y no ser posible su detección. Esta concentración se realiza generalmente mediante extracción de la fracción aromática del destilado, desecando el extracto y concentrando como se verá en el apartado de extracción.

La destilación con distintas variantes se ha aplicado a gran cantidad de alimentos diferentes, por destilación en corriente de vapor, quesos (Ceccon, 1981 y 1986), arándanos (Horvat, 1983 y 1985), algas comestibles (Kajiwara, 1988), loquats (Shaw, 1982), tes (Hazarika, 1984), etc. y por destilación al vacío albaricoques (Chairote, 1981) (Guichard, 1988), lecitina de soja (Kim, 1984), agua (Kozloski, 1985), mangos (Sakho, 1985), jugo de fruta de la pasión (Kuo, 1985), aceite de manteca (Nawar, 1988), almejas (Yasuhara, 1987), nectarinas (Takeoka, 1988), kiwis (Young, 1983), feijoas (Shaw, 1990), ciruelas (Ismail, 1981), etc.

\section{Extracción}

En la mayoría de los alimentos es necesario realizar una destilación previa a la extracción ya que junto con los volátiles pueden ser extraídas otra serie de sustancias no volátiles que pueden interferir el análisis, sin embargo, cierto tipo de alimentos como los zumos de frutas no presentan este inconveniente y pueden someterse a una extracción directa (Alberola, 1979b).

Es una técnica muy simple que consiste en la separación de la fracción aromática del alimento con un disolvente adecuado. Puede realizarse de forma manual o con extractores líquido-líquido. Presenta el inconveniente de la posibilidad de formación de emulsiones entre el disolvente y el alimento (Mateos, 1990).

Una vez finalizada la extracción se suele eliminar el agua del extracto con sulfato sódico anhidro y, aunque a veces no es necesario concentrar (Kok, 1987), generalmente se hace necesaria esta etapa que puede realizarse utilizando corriente de nitrógeno o aire (Lanza, 1976), empleando columnas Vigreaux (Loyola, 1988) o en rotavapor.

El disolvente utilizado debe ser lo más selectivo posible y tener alto poder de extracción para que el extracto tenga la mayor cantidad posible de componentes del aroma y la mínima de contaminantes. Se han utilizado diferentes disolventes siendo de interés los de bajo punto de ebullición por no ser necesario aumentar la temperatura para concentrar. Asimismo, se han estudiado técnicas de inyección para conseguir la máxima eliminación de disolvente con la mínima de volátiles (Herraiz, 1987) (Herraiz, 1989).

El método de extracción se ha aplicado a muestras de vino de Jerez (Cabezudo, 1988), cítricos (Pino, 1988), nectarinas (Engel, 1988), pisco (bebida chilena) (Loyola, 1988), jarabe de abedul (Kallio, 1989), guayaba (Nishimura, 1989), vinos (Moret, 1984) (Baumes, 1986) y zumo de uvas (Blanch, 1991) entre otras.

\section{Destilación-Extracción Simultánea}

Es un método ampliamente utilizado que fue introducido por Likens y Nickerson en 1964 (Rijks, 1983). Consiste en que la muestra diluida en agua y el disolvente destilan de forma separada y condensan en la misma zona produciéndose en ella la extracción, separándose y recirculando posteriormente las dos fases. De esta técnica original se han descrito numerosas modificaciones (Maarse, 1970) (Schultz, 1977) (Auyeng, 1981), entre ellas una destinada a la obtención de volátiles de grasas y aceites donde se obtienen condensados libres de agua (Weurman, 1969), y una microversión del aparato original para aplicaciones analíticas (Godefroot, 1981).

El método presenta ventajas como usar pequeñas cantidades de disolvente, introduciéndose por ello pocos contaminantes, poder obtener una concentración alta de volátiles en poco tiempo y poder minimizar la degradación térmica trabajando a presión reducida (Mateos, 1990). También presenta inconvenientes como el de no ser adecuada para volátiles termolábiles.

Este método se ha aplicado a muchos alimentos: quesos (Aishima, 1987), setas (Chen, 1984), peras 
(Russell, 1981), jugo de pomelo (Núñez, 1985), pepino (Shiota, 1988), pericarpo de melón (Horvat, 1987), uvas (Horvat, 1984), pistachos (Wyllie, 1990), tes (Owuor, 1990), etc.

Sin embargo, a pesar de la notable aplicación de estos métodos a distintos alimentos no es tan amplia su aplicación a muestras de aceite, siendo escasos los trabajos encontrados en los últimos años.

Recientemente se ha desarrollado un método de destilación a vacío con recogida de los volátiles en tres trampas frías (enfriadas dos con $\mathrm{N}_{2}$ líquido y una con acetona/ $\mathrm{CO}_{2}$ sólido), posterior disolución con éter etílico y finalmente concentración por destilación y microdestilación (Ullrich, 1988). Se aplica al estudio de la alteración del flavor del aceite de soja identificando los compuestos de olor intenso que se forman durante la reversión del flavor. Otro procedimiento utilizado consiste en una codestilación a vacío del aceite con éter etílico y recogida en tres trampas enfriadas con $\mathrm{N}_{2}$ líquido y posteriormente en un "dedo frío" enfriado con agua corriente, procediendo a la extracción de este último con éter y uniendo a los extractos etéreos recogidos en las trampas frías, por último se destila y microdestila para concentrar (Guth, 1989). Se ha aplicado al estudio de uno de los compuestos responsables de la reversión del flavor del aceite de soja.

Los datos correspondientes a estas muestras se encuentran recogidos en la Tabla III.

Tabla III

\section{Parámetros y referencias de los métodos de destilación}

\begin{tabular}{|c|c|c|c|c|c|c|}
\hline $\begin{array}{l}\text { Cantidad } \\
\text { de muestra }\end{array}$ & $\begin{array}{l}\text { Dest } \\
T(P)\end{array}$ & $\begin{array}{l}\text { stilación } \\
P \text { (mbar) }\end{array}$ & Disolvente & Columna & $\begin{array}{l}\text { Gas } \\
\text { portador }\end{array}$ & Referencia \\
\hline $\begin{array}{l}2,25 \mathrm{Kg} \\
\text { (soja) }\end{array}$ & 50 & $3 \times 10^{-2}$ & Eter etílico & $\begin{array}{c}\text { SE-30 } \\
\text { OV-1701 }\end{array}$ & $\mathrm{He}$ & Ullich, 1988 \\
\hline $\begin{array}{l}2 \mathrm{Kg} \\
\text { (soja) }\end{array}$ & 34 & $6 \times 10^{-5}$ & Eter etílico & $\begin{array}{c}\text { SE-30 } \\
\text { OV-1701 } \\
\text { SE-54 } \\
\text { SW-10 }\end{array}$ & $\mathrm{He}$ & Guth, 1989 \\
\hline
\end{tabular}

\subsection{Espacio de cabeza dinámico}

La técnica fue introducida por Swinnerton et al. (1962a y b) y aplicada a la determinación de gases disueltos en muestras acuosas. Consiste en arrastrar los volátiles de la muestra, sometida a una temperatura determinada, con un gas inerte a un flujo controlado y pasarlos a través de una trampa donde quedan retenidos para posteriormente ser desorbidos e inyectados en el cromatógrafo para su separación.

Se pueden distinguir dos modalidades dentro de esta técnica (Núñez, 1984):

a) la primera de ellas sería el espacio de cabeza dinámico propiamente dicho, que consiste en barrer la superficie de la muestra sometida a agitación con el gas inerte.

b) la segunda, conocida como técnica de arrastreatrape (purgue and trap), consiste en hacer borbotear el gas a través de la muestra, pudiéndose aplicar de dos formas diferentes: en circuito abierto, donde el gas pasa por la muestra y la trampa y de aquí al exterior o en circuito cerrado, donde el gas se recicla a través de la muestra y la trampa.

Existen numerosos factores que afectan al proceso tales como diámetro y longitud de las trampas, forma y tamaño del recipiente usado en el aislamiento, tamaño de partícula del adsorbente (Murray, 1977) (Ramstad, 1980) (Krost, 1982) (Senf, 1990), pero las tres variables fundamentales que lo controlan son temperatura, tiempo y flujo de arrastre; estas dos últimas deben ser fijadas previamente, teniendo en cuenta que valores demasiado bajos pueden conducir a un arrastre defectuoso y por el contrario valores demasiado altos a pérdida de volátiles. La temperatura (Mordret, 1985) está condicionada por el tipo de compuestos que se deseen analizar, ya que si se trabaja a baja temperatura (no más de $60-70^{\circ} \mathrm{C}$ ) los volátiles recuperados corresponden a los realmente presentes en la muestra en el momento del análisis, con lo cual las cantidades son muy pequeñas y el análisis es delicado. Si se utilizan temperaturas más elevadas (hasta $160^{\circ} \mathrm{C}$ ) los compuestos obtenidos corresponden no sólo a los realmente presentes en la muestra, sino también pueden aparecer compuestos volátiles formados por degradación térmica de algunos precursores, en este caso las cantidades recuperadas son mayores y el análisis se puede realizar con más facilidad.

En las muestras gaseosas obtenidas con la utilización de este método (Núñez, 1984) suele haber una cantidad reducida de sustancias volátiles, debido a que están constituidas fundamentalmente por gas de arrastre y vapor de agua. Por ello, se hace necesaria una etapa de concentración que se realiza mediante el uso de $A$ ) trampas de materiales adsorbentes y $B$ ) trampas criogénicas.

A) Las trampas adsorbentes están constituidas por un tubo de vidrio o acero inoxidable relleno de una cantidad determinada de material adsorbente (trampa), de manera que al pasar el gas inerte cargado de volátiles a través de ella estos últimos quedan retenidos.

Son muy numerosos los materiales que se han empleado con este fin (Clark, 1975) (Dressler, 1979) (Crisp, 1980):

a) Carbón activo y materiales grafitizados: Han sido los más utilizados en los primeros trabajos de este tipo. Presentan una gran superficie específica y alta capacidad de adsorción, siendo muy adecuados para desorción con disolventes, pero no para desorción térmica porque los compuestos son retenidos muy fuertemente en su superficie y se necesitarian temperaturas muy elevadas para obtener una desorción cuantitativa (Drozd, 1979). Esto unido a que presentan alta afinidad por el agua $y$, en algunos casos adsorción selectiva, ha hecho que en los últimos años se haya preferido el uso de polímeros 
porosos que tienen la ventaja de retener poca agua y no presentar adsorción irreversible, aunque soportan temperaturas menos elevadas.

b) Serie Tenax: A pesar de que posee una superficie específica pequeña, presenta una buena capacidad de adsorción para muchos compuestos y una alta estabilidad térmica que permite la desorción de sustratos a alta temperatura (hasta $350^{\circ} \mathrm{C}$ ) sin aparición de artefactos. Retiene poca agua y es adecuado para componentes de medio y alto punto de ebullición. Actualmente es el polímero más utilizado.

c) Serie Porapak: Poseen menor capacidad de adsorción que el carbón activo y mayor que el Tenax, algunos presentan selectividad por cierto tipo de compuestos. La estabilidad térmica es baja.

d) Serie Chromosorb: Algunos de ellos poseen una adecuada superficie específica, sus propiedades son semejantes al Tenax pero presentan menor estabilidad térmica.

e) Serie Amberlite: Tienen alta superficie especifica pero presentan inestabilidad térmica, por lo que se han empleado realizando desorción con disolventes.

f) Otros: Materiales adsorbentes tales como el Ostion SP-1, Synachrom, Spheron, etc. han sido mucho menos utilizados como material de atrape.

Todas estas trampas necesitan un acondicionamiento del material para evitar interferencias en el análisis. Según el tipo de adsorbente se aconseja un acondicionamiento determinado (Lewis, 1980) (Betti, 1985) (MacLeod, 1986) (Riba, 1988).

B) Las trampas criogénicas están constituidas por un trozo de columna o un tubo capilar de otro tipo que se enfría a temperatura muy baja, generalmente con nitrógeno líquido, de tal forma que los compuestos que pasan a través de ella condensan en su interior. La desorción se realiza pasando un flujo de gas inerte $y$ elevando la temperatura, con lo que los volátiles pasan directamente a la columna.

En la bibliografía se encuentran casos en los que se utilizan los dos tipos de trampas a la vez, realizándose primero una concentración sobre un polímero y pasando después los volátiles por una trampa fría donde condensan (Werkhoff, 1987a y b).

La desorción de los volátiles una vez atrapados suele realizarse por dos procedimientos: 1) desorción con disolventes o 2) desorción térmica.

1) Desorción con disolventes: este procedimiento se realiza con un pequeño volumen de disolvente orgánico y de manera que el coeficiente de reparto esté a favor del eluyente. La cantidad de disolvente no debe ser demasiado baja pues podría producirse una desorción incompleta, ni demasiado alta porque podrían quedar los volátiles muy diluidos y sería necesario concentrar, soliendo realizarse por evaporación, lo cual tiene inconvenientes tales como: a) la introducción de artefactos en la muestra, b) el pico del disolvente puede enmascarar parte de la misma y c) el método no es rápido y pueden producirse pérdidas al evaporar (Núñez, 1984).

Esta técnica presenta la ventaja de que se pueden realizar varias inyecciones de la misma muestra y ello hace el análisis más exacto.

2) Desorción térmica: Este procedimiento está siendo cada vez más utilizado en los últimos años (Burger, 1986) (Selke, 1987) (Raghavan, 1989) (Bishop, 1990) (Dubrova, 1990) a pesar de sus inconvenientes (descomposición térmica o impurezas de adsorbente). Consiste en calentar la trampa a temperatura elevada pasándole un flujo de gas inerte en sentido contrario a aquél en el que se realizó la adsorción. Se han utilizado distintos dispositivos para conseguir el calentamiento, algunos adosados al inyector y otros realizando una modificación del mismo. El principal inconveniente del método es que cada muestra puede ser inyectada una sola vez y en caso de necesitar repetir la inyección hay que realizar todo el proceso.

El espacio de cabeza dinámico ha sido profusamente aplicado al análisis del flavor de diferentes tipos de alimentos durante los últimos años: leche (Urbach, 1987), nectarinas (Takeoka, 1988), feijoas (Shaw, 1983), carnes de cerdo y buey (Mottram, 1982), champán (Loyaux, 1981), extracto de malta (Farley, 1980), trufas (Talou, 1989), grasa ovina (Suzuki, 1985), mangos (Bartley, 1987) kiwis (Young, 1985), plátanos (Macku, 1987), melocotones (Narain, 1990), babacos (Bartley, 1988), colas de cangrejo (Vejaphan, 1988), zumo de uvas (Blanch, 1991), etc.

Con referencia a aceites comestibles, ha habido en los últimos años un aumento de la utilización de esta técnica de recogida de volátiles. El atrape sobre trampas de carbón activo con desorción con $\mathrm{S}_{2} \mathrm{C}$ como disolvente fue muy usado en los años 70 y 80 para estudiar los volátiles presentes en aceite de oliva virgen. En estos trabajos (Olías, 1977, 1978 y 1980) (Dobarganes, 1980) se describen diferentes dispositivos de recogida, se parte de grandes cantidades de muestra y se emplean largos períodos de tiempo para el arrastre. En algunos de ellos (Dobarganes, 1980) (Olías, 1980) (Gutiérrez, 1981) se utiliza en lugar de una sola trampa, tres trampas de carbón activo en serie, de tal forma que las dos primeras se extraen juntas con $\mathrm{S}_{2} \mathrm{C}$ y constituyen el concentrado principal, y la tercera, que se extrae aparte, sirve para comprobar que los volátiles se encuentran en las dos primeras y para prevenir que en aceites muy aromáticos se pudieran llegar a saturar las dos primeras. La mayor parte de estos trabajos están orientados hacia la identificación de los diferentes volátiles presentes en el aceite.

También se ha aplicado la técnica de atrape sobre carbón activo y desorción con sulfuro de carbono en estudios de termoxidación de aceites de distinto tipo (Dobarganes, 1986).

El empleo de otro disolvente como es el éter etílico en la desorción de las trampas de carbón activo ha sido utilizado en varios trabajos en los que se investigan los compuestos volátiles responsables de la rancidez del aceite de oliva virgen (Solinas, 1985 y 1987a), en el estudio de volátiles relacionados con variedad (Solinas, 1988a), en la evolución de algunos componentes del aroma en relación con el grado de maduración de la aceituna (Solinas, 1987b), y también en la determinación de compuestos volátiles producidos en aceites oxidados du- 
rante el proceso de fritura comparando otros aceites vegetales con el aceite de oliva (Solinas, 1988b).

El procedimiento de desorción térmica es el más utilizado recientemente, realizándose el atrape de volátiles sobre distintas trampas. El estudio de identificación de compuestos volátiles de 8 tipos de aceite a diferentes niveles de oxidación (Snyder, 1985) se ha realizado sobre trampa de Tenax, desorbiendo a $180^{\circ} \mathrm{C}$ sobre columna enfriada a $-50^{\circ} \mathrm{C}$. Se han estudiado aceites de oliva y girasol obteniéndose una serie de cromatogramas "huella" característicos de cada tipo de aceite (Gasparoli, 1987) realizando la concentración en una trampa fría $\left(-120^{\circ} \mathrm{C}\right)$ y desorbiendo a $200^{\circ} \mathrm{C}$.

La desorción térmica de trampas de Tenax $\left(170^{\circ} \mathrm{C}\right)$ también se aplicó a la determinación de volátiles presentes en aceite de soja sometiéndolos a diferentes temperaturas durante el proceso de aislamiento y concentración y demostrándose la influencia de esta variable en los resultados obtenidos (Selke, 1987). Asimismo se ha utilizado el atrape sobre Tenax GC y desorción térmica $\left(220^{\circ} \mathrm{C}\right)$ en un trabajo sobre aceite de soja que realiza una comparación de los tres métodos más utilizados: inyección directa, espacio de cabeza estático y dinámico, apreciándose ventajas e inconvenientes en cada uno de ellos (Snyder, 1988).

Una variante del método consiste en recoger los volátiles sobre una trampa de Tenax que posteriormente es desorbida térmicamente sobre otra menor y después reconcentrada en una tercera desde la que son inyectados en el cromatógrafo, el uso de esta técnica en el análisis de la calidad del flavor de aceites de soja y maíz permite una disminución del tiempo de análisis y muestra buenas correlaciones con el procedimiento de evaluación sensorial (Raghavan, 1989).

Otras trampas utilizadas son las que llevan distintos materiales adsorbentes, una trampa de bolas de vidrio (para atrapar los compuestos de alto punto de ebullición) / Tenax (compuestos de PE intermedio) / Ambersorb (compuestos muy volátiles) ha sido empleada en el análisis de aceite de soja almacenado, en este caso la desorción se realiza térmicamente a $250^{\circ} \mathrm{C}$ (Wyatt, 1987).

En la Tabla IV se encuentran recogidos los trabajos que utilizan estas técnicas de espacio de cabeza dinámico y arrastre-atrape. Puede apreciarse que se han utilizado distintas modalidades, siendo de uso más reciente el atrape sobre Tenax y la desorción térmica.

Tabla IV

Parámetros y referencias de los métodos de espacio de cabeza dinámico

\begin{tabular}{|c|c|c|c|c|c|c|c|}
\hline \multirow{2}{*}{$\begin{array}{c}\text { Cantidad de } \\
\text { muestra }\end{array}$} & \multicolumn{3}{|c|}{ Arrastre } & \multirow{2}{*}{\multicolumn{2}{|c|}{ Trampa Desorción }} & \multirow[t]{2}{*}{ Columna } & \multirow[t]{2}{*}{ Referencia } \\
\hline & $\mathrm{T}^{\mathrm{a}}\left({ }^{\circ} \mathrm{C}\right)$ & $t(h)$ & Gas & & & & \\
\hline $\begin{array}{l}250 \mathrm{ml} \\
\text { (oliva virgen) }\end{array}$ & 65 & 48 & $\mathrm{~N}_{2}$ & $\mathrm{C}$ activo & $\mathrm{S}_{2} \mathrm{C}$ & $\begin{array}{l}30 \% \text { Carbowax } 20 \mathrm{M} \\
\text { Chromosorb } 80 / 100\end{array}$ & Olias, 1977 \\
\hline $\begin{array}{l}250 \mathrm{ml} \\
\text { (oliva virgen) }\end{array}$ & 65 & 48 & $\mathrm{~N}_{2}$ & C activo & $\mathrm{S}_{2} \mathrm{C}$ & $\begin{array}{l}30 \% \text { Carbowax } 20 \mathrm{M} \\
\text { Chromosorb } 100 / 120\end{array}$ & Olías, 1978 \\
\hline $\begin{array}{l}1.000 \mathrm{ml} \\
\text { (oliva virgen) }\end{array}$ & 80 & 48 & $\mathrm{~N}_{2}$ & C activo & $\mathrm{S}_{2} \mathrm{C}$ & $\begin{array}{l}5 \% \text { Carbowax } 20 \mathrm{M} \\
\text { Chromosorb } 80 / 100\end{array}$ & $\begin{array}{l}\text { Dobarganes, } 1980 \\
\text { Olías, } 1980 \\
\text { Gutiérrez, } 1981\end{array}$ \\
\hline $\begin{array}{l}100 \mathrm{ml} \\
\text { (oliva virgen) }\end{array}$ & 37 & 5 & $\mathrm{~N}_{2}$ & C activo & $\begin{array}{l}\text { Eter } \\
\text { Etílico }\end{array}$ & Carbowax $20 \mathrm{M}$ & Solinas, 1985 \\
\hline $\begin{array}{l}5 \mathrm{~g} \\
\text { (oliva, girasol, } \\
\text { algodón, etc.) }\end{array}$ & 170 & 0,16 & $\mathrm{He}$ & Tenax & $\begin{array}{l}\text { Térmica } \\
\left(180^{\circ} \mathrm{C}\right)\end{array}$ & Durabond-5 & Snyder, 1985 \\
\hline $\begin{array}{l}250 \mathrm{~g} \\
\text { (palma, oliva, } \\
\text { soja, girasol) }\end{array}$ & 140 & 6 & $\mathrm{~N}_{2}$ & C activo & $\mathrm{S}_{2} \mathrm{C}$ & OV1/OV101 BP & Dobarganes, 1986 \\
\hline (oliva, girasol) & - & - & $\mathrm{He}$ & Fría & $\begin{array}{l}\text { Térmica } \\
\left(200^{\circ} \mathrm{C}\right)\end{array}$ & SE-30 & Gasparoli, 1987 \\
\hline $\begin{array}{l}\text { (oliva virgen, } \\
\text { girasol, soja, etc.) }\end{array}$ & 37 & 3 & $\mathrm{~N}_{2}$ & C activo & $\begin{array}{l}\text { Eter } \\
\text { Etílico }\end{array}$ & Carbowax 20M & $\begin{array}{l}\text { Solinas, } 1987 a \text { y b } \\
\text { Solinas, 1988a y b }\end{array}$ \\
\hline $\begin{array}{l}0,5 \mathrm{~g} \\
\text { (soja) }\end{array}$ & 100 & 0,08 & $\mathrm{~N}_{2}$ & $\begin{array}{c}\text { Vidrio/ } \\
\text { Tenax/ } \\
\text { Ambersorb }\end{array}$ & $\begin{array}{l}\text { Térmica } \\
\left(250^{\circ} \mathrm{C}\right)\end{array}$ & Durabond-5 & Wyatt, 1987 \\
\hline $\begin{array}{l}5 \mathrm{ml} \\
\text { (soja) }\end{array}$ & $60-180$ & 0,25 & $\mathrm{He}$ & Tenax & $\begin{array}{l}\text { Térmica } \\
\left(170^{\circ} \mathrm{C}\right)\end{array}$ & Durabond-5 & Selke, 1987 \\
\hline $\begin{array}{l}5 \mathrm{~g} \\
\text { (soja) }\end{array}$ & 180 & 0,25 & $\mathrm{He}$ & Tenax & $\begin{array}{l}\text { Térmica } \\
\left(220^{\circ} \mathrm{C}\right)\end{array}$ & Durabond-5 & Snyder, 1988 \\
\hline $\begin{array}{l}1 \mathrm{~g} \\
\text { (soja, maíz) }\end{array}$ & 150 & 0,33 & $\mathrm{~N}_{2}$ & Tenax & $\begin{array}{l}\text { Térmica } \\
\left(250^{\circ} \mathrm{C}\right)\end{array}$ & $D X-1$ & Raghavan, 1989 \\
\hline
\end{tabular}


Aunque esta técnica presenta inconvenientes, tiene la ventaja de poder aplicarse a muy diversas cantidades de muestra y en un amplio rango de temperaturas, lo cual unido a la concentración de volátiles en las trampas adecuadas permite un aumento de la sensibilidad, además de la posibilidad de estudiar los volátiles realmente presentes en la muestra en el momento del análisis por ser posible trabajar a bajas temperaturas.

\section{BIBLIOGRAFIA}

Aishima, T. y Nakai, S. (1987).- "Pattern recognition of GC profiles for classification of cheese variety".- J. Food Sci. 52, 939-942.

Alberola, J. e Izquierdo, L. (1979a).- "La fracción aromática del zumo de naranja. II. Análisis del espacio de cabeza".- Rev. Agroquim. Tecnol. Aliment. 19, 327-337.

Alberola, J. e Izquierdo, L. (1979b).- "La fracción aromática del zumo de naranja. III. Análisis de volátiles totales".- Rev. Agroquím. Tecnol. Aliment. 19, 469-482.

Auyeng, C. Y. y MacLeod, A. J. (1981).- "A comparison of the "incy of the Lickens and Nickerson extractor for aqueous, lipid/aq. $\therefore$, and lipid samples".- J. Agric. Food Chem. 29, 502-505.

Bartley, J. P. y Schwede, A. (1987).- "Volatile flavor components in the headspace of the Australian or "Bowen" Mango".- J. Food Sci. 52 353-355.

Bartley, J. P. (1988).- "Volatile flavor components in the headspace of the babaco fruit (Carica Pentagonia).- J. Food Sci. 53, 138-140.

Baumes, R.; Cordonnier, R.; Nitz, S. y Drawert, F. (1986).- "Identification and determination of volatile constituents in wines from different vine cultivars".- J. Sci. Food Agric. 37, 927-943.

Benkler, K. F. y Reineccius, G. A. (1980).- "Flavor isolation from fatty foods via solvent extraction and membrane dialysis".- J. Food Sci. 45, 1084-1085.

Betti, A.; Coppi, S. y Bighi, C. (1985).-- "Pre-concentration of organic pollutants. Potential interference from the use of styrene copolymer adsorbents".- J. Chromtogr. 349, 181-187.

Bishop, R. W. y Valis, R. J. (1990).- "A laboratory evaluation of sorbent tubes for use with a thermal desorption gas chromatography-mass selective detection technique".- J. Chromatogr. Sci. 28, 589-593.

Blanch, G. P.; Reglero, G.; Herraiz, M. y Tabera, J. (1991).- "A comparison of different extraction methods for the volatile components of grape juice".- J. Chromatogr. Sci. 29, 11-15

Booker, J. L. (1985).- "Collecting volatile compounds by simple diffusion: an alternative to purge-and-trap".- J. Chromatogr. Sci. 23, 415-416

Burger, B. V. y Munro, Z. (1986).- "Headspace gas analysis. Quantitative trapping and thermal desorption of volatiles using fused-silica open tubular capillary traps".- J. Chromatogr. 370, 449-464.

Cabezudo, M. D. y Almy, J. (1988).- "Enrichment of aldehydes and ketones in sherry".- J. Food Sci. 53, 1900-1901, 1904

Ceccon, L.; Stancher, B. y Calzolari, C. (1981).- "La frazione lipida nella caratterizzazione dei formaggi. Acidi grassi liberi volatili".- Riv. Ital. Sostanze Grasse 58, 132-138.

Ceccon, L. (1986).- "La frazione lipidica nella caratterizzazione merceologica dei formaggi. Acidi grassi liberi volatili. Nota IV".- Riv. Ital. Sostanze Grasse 63, 551-554.

Clark, R. G. y Cronin, D. A. (1975).- "The use of activated charcoal for the concentration and analysis of headspace vapours containing food aroma volatiles".- J. Sci. Food Agric. 26, 1615-1624

Crisp, S. (1980).- "Solid sorbent gas samplers".- Ann. occup. Hyg. 23, 47 76.

Chairote, G.; Rodriguez, F. y Crouzet, J. (1981).- "Characterization of additional volatile flavor components of apricot". J. Food Sci. 46, 1898-1901, 1906

Chen, Chu-Chin y Wu, Chung-May (1984).- "Volatile components of mushrrom (Agaricus subrufecens)".- J. Food Sci. 49, 1208-1209.

Chialva, F.; Doglia, G.; Gabri, G. y Ulian, F. (1983).- "Direct headspace gas chromatographic analysis with glass capillary columns in quality control of aromatic herbs".- J. Chromatogr. 279, 333-340.

Davis, P. L. (1970).- "A simple method to prevent loss of volatile during headspace analysis".- J. Chromatogr. Sci. 8, 423-424.

Del Barrio, A.; Gutiérrez, F. y Gutiérrez, R. (1981).- "Aplicación de la cromatografla gas-líquido, técnica de espacio de cabeza, al problema del atrojado de los aceites de oliva. I".- Grasas y Aceites 32, 155 161.
Del Barrio, A.; Gutiérrez, F.; Cabrera, J. y Gutiérrez, R. (1983).- "Aplicación de la cromatografia gas-líquido, técnica de espacio de cabeza, al problema del atrojado de los aceites de oliva. II".- Grasas y Aceites 34, 1-6.

Dobarganes, M. C.: Olias, J. M. y Gutiérrez, R. (1980).- "Componentes volátiles en el aroma del aceite de oliva virgen. III. Reproducibilidad del método utilizado para su aislamiento, concentración y separación".- Grasas y Aceites 31, 317-321.

Dobarganes, M. C.; Ríos, J. J. y Pérez-Camino, M. C. (1986).-" Relaciones entre la composición de aceites vegetales y los componentes volátiles producidos durante su termoxidación".- Grasas y Aceites 37 61-67.

Dressler, M. (1979).- "Extraction of trace amounts of organic compounds from water with porous organic polymers".- J. Chromatogr. 165, 167206.

Drozd, J. y Novák, J. (1979).- "Headspace gas analysis by gas chromatography".- J. Chromatogr. 165, 141-165.

Dubrova, M. A.; Mindlin, L. O.; Shchedrina, M. M.; Belfer, A. G.; Nikitina M. A. y Rudenko, B. A. (1990).- "Headspace gas chromatography with open-tubular columns for determining the evaporation rate of essential oils, perfumery compositions and perfumes".- J. Chromatogr. 520, 169-174.

Dupuy, H. P.; Fore, S. P. y Goldblatt, L. A. (1971).- "Elution and analysis of volatiles in vegetable oils by gas chromatography".- J. Am. Oil Chemists' Soc. 48,876

Dupuy, H. P.; Fore, S. P. y Goldblatt, L. A. (1973).- "Direct gas chromatographic examination of volatiles in salad oil and shortenings".J. Am. Oil Chemists' Soc. 50, 340-342.

Dupuy, H. P.; Flick Jr., G. J.; Bailey, M. E.; St. Angelo, A. J.; Legendre, M G. y Sumrell, G. (1985).- "Direct sampling capillary gas chromatography of volatiles in vegetable oils".- J. Am. Oil Chemists' Soc. 62, 16901693.

Engel, K. H.; Flath, R. A.; Buttery, R. G.; Mon, T. R.; Ramming, D. W. y Teranishi, R. (1988).- "Investigation of volatile constituents in nectarines. 1. Analytical and sensory characterization of aroma components in some nectarine cultivars".- J. Agric. Food Chem. 36 549-553.

Farley, D. R. y Nursten, H. E. (1980).- "Volatile flavour components of malt extract".- J. Sci. Food Agric. 31, 386-396.

Flath, R. A.; Sugisawa, H. y Teranishi, R. (1981).- "Problems in flavor research" en "Flavour Research. Recent advances".- Teranishi, R. Flath, R. A. y Sugisawa, H. (Ed.).- Marcel Dekker Inc., Nueva York.

Gasparoli, A.; Fedeli, E. y Manganiello, B. (1986).- "Olio vergine di oliva: valutazione dei caratteri organolettici attraverso tecniche strumentali". Riv. Ital. Sostanze Grasse 63, 571-582.

Gasparoli, A. y Fedeli, E. (1987).- "Valutazione dei componenti volatil negli oli alimentari: un approccio alla tecnica "Purgue and Trap".- Riv. Ital. Sostanze Grasse 64, 453-460.

Gensic, J. L.; Szuhaj, B. F. y Endres, J. G. (1984).- "Automated gas chromatographic system for volatile profile analysis of fats and oils". J. Am. Oil Chemists' Soc. 61, 1246-1249.

Godefroot, M.; Sandra, P. y Verzele, H. (1981).- "New method fo quantitative essential oil analysis".- J. Chromatogr. 203, 325-335.

Golovnya, R. V. (1982).- "Some analytical problems in flavour research". J. Chromatogr. 251, 249-264.

Guichard, E. (1988).- "Quantification of some volatile aromatic compounds of apricot by adding standards".- J. Food Sci. 53, 1902-1904.

Guth, H. y Grosch, W. (1989).- "3-Methylnonane-2, 4-dione -An intense odour compound formed during flavour reversion of soyabean oil". Fat Sci. Technol. 91, 225-230.

Gutiérrez, R.: Olias, J. M. Gutiérrez, F. Cabrera, J. y Del Barrio, A. (1975).- "Los métodos organolépticos y cromatográficos en la valoración de las características aromáticas del aceite de oliva virgen". Grasas y Aceites 26, 21-31.

Gutiérrez, R.; Dobarganes, M. C.: Gutiérrez, F. y Olias, J. M. (1981)."Componentes volátiles en el aroma del aceite de oliva virgen. V. Aceites obtenidos de frutos atrojados".- Grasas y Aceites 32, 299 303.

Hawthorne, S. B.; Krieger, M. S. y Miller, D. J. (1988).- "Analysis of flavor and fragance compounds using supercritical fluid extraction coupled with gas chromatography".- Anal. Chem. 60, 472-477.

Hazarika, M.: Mahanta, P. K. y Takeo, T. (1984).- "Studies on some volatile flavour constituents in orthodox black teas of various clones and flushes in North-east india".- J. Sci. Food Agric. 35, 1201-1207.

Herraiz, M.; Reglero, G.; Loyola, E. y Herraiz, T. (1987).- "Sampling of volatile components using a PTV in the solvent split mode".- J. High Resolut. Chromatogr. Chromatogr. Commun. 10, 598-602.

Herraiz, M.; Reglero, G. y Herraiz, T. (1989).- "Evaluation of a PTV injector 
for quantitative analysis of volatile compounds at low concentrations".J. High Resolut. Chromatogr. 12, 443-446.

Horvat, R. J.; Senter,. S. D. y Dekazos, E. D. (1983).- "GLC-MS Analysis of volatile constituents in rabbiteye blueberries".- J. Food Sci. 48 278-279.

Horvat, R. J. y Senter, S. D. (1984).- "Identification of the volatile constituents from scruppernong berries (Vitis rotundifolia)".- J. Food Sci. 49, 64-66, 81.

Horvat, R J y Senter, S. D. (1985) - "Comparison of the volatile constituents from rabbiteye blueberries (Vaccinium ashei) during ripening".- J. Food Sci. 50, 429-431.

Horvat, R. J. y Senter, S. D. (1987).- "Identification of additional volatile compounds from cantaloupe".- J. Food Sci. 52, 1097-1098.

Ismail, H. M.; Williams, A. A. y Tucknott, O. G. (1981).- "The flavour of plums (Prunus domestica L.). An examination of the aroma components of plum juice from the cultivar Victoria".- J. Sci. Food Agric. 32, 613-619.

Jackson, H. W. y Giacherio, D. J. (1977).- "Volatiles and oil quality".- J. Am. Oil Chemists' Soc. 54, 458-460.

Jackson, H. W. (1981).- "Techniques for flavor and odor evaluation of soy oil".- J. Am. Oil Chemists' Soc. 58, 227-231.

Jennings, W. G. y Filsoof, M. (1977).- "Comparison of sample preparation techniques for gas chromatographic analysis".- J. Agric. Food Chem. $25,440-445$

Kajiwara, T.; Hatanaka, A.; Kawai, T.; Ishihara, M. y Tsuneya, T. (1988)."Study of flavor compounds of essential oil extracts from edible Japanese kelps".- J. Food Sci. 53, 960-962.

Kallio, H. (1989).- "Aroma of birch syrup".- J. Agric. Food Chem. 37, 13671371.

Kim, Heasook, Ho, Chi-Tang y Chang, Stephen S. (1984).- "Isolation and identification of volatile flavor compounds in commercial oil-free soybean lecitin".- J. Am. Oil Chemists' Soc. 61, 1235-1238.

Kok, M. F.; Yong, F. M. y Lim, G. (1987).- "Rapid extraction method for reproducible analysis of aroma volatiles".- J. Agric Food Chem. 35 779-781.

Kozloski, R. P. (1985).- "Simple method for concentrating volatiles in water for gas chromatographic analysis by vacuum distillation".- J Chromatogr. 346, 408-412.

Krost, K. J.; Pellizari, E. D.; Walburn, S. G. y Hubbard, S. A. (1982) "Collection and analysis of hazardous organic emissions".- Anal. Chem. 54, 810-817.

Kuo, May-Chien, Chen, Su-Lin, Wu, Chung-May y Chen, Chu-Chin (1985)."Changes in volatile components of Passion fruit juice as affected by centrifugation and pasteurization".- J. Food Sci. 50, 1208-1210.

Lanza, E.; Ko, K. H. y Palmer, J. K. (1976).- "Aroma production by cultures of Ceratocystis moniliformis".- J. Agric. Food Chem. 24, 1247-1250.

Lewis, M. J. y Williams, A. A. (1980).- "Potential artefacts from using porous polymers for collecting aroma components".- J. Sci. Food Agric. 31, 1017-1026.

Loyaux, D.; Roger, S. y Adda, J. (1981).- "The evolution of champagne volatiles during ageing".- J. Sci. Food Agric. 32, 1254-1258.

Loyola, E.; Almy, J.; Martin-Alvarez, P. y Cabezudo, M. D. (1988).- "The Flavor of Chilean Pisco".- Frontiers of Flavor, Proccedings of the 5 th International Flavor Conference, Porto Karras, Chalkidiki, Greece, 729-742.

Maarse, H. y Kepner, R. E. (1970).- "Changes in composition of volatile terpenes in douglas fir needles during maturation".- J. Agric. Food Chem. 18, 1095-1101.

Macku, C. y Jennings, W. G. (1987).- "Production of volatiles by ripening bananas".- J. Agric. Food Chem. 35, 845-848

MacLeod, G. y Ames, J. M. (1986).- "Comparative assessment of the artefact background on thermal desorption of Tenax GC and Tenax TA".- J. Chromatogr. 355, 393-398

Mariani, C.; Venturini, S. y Fedeli, E. (1990).- "Sulla presenza di prodotti alogenati volatili negli oli vergini di oliva".- Riv. Ital. Sostanze Grasse 67, 239-244.

Mateos, A. y Carbonell, E. (1990).- "Análisis de la fracción aromática de alimentos. Técnicas de extracción y concentración".- Rev. Agroquim. Tecnol. Aliment. 30, 431-444.

Mehlitz, A. y Gierschner, K. (1962).- "Ueber bisherige gaschromatographische Untersuchungen der Aromastoffe von Früchten".- Inter. Fed. Fruit Juice Producers, Symposium Volatile Fruit Flavours, Bern, 25

Min, D. B. (1981).- "Correlation of sensory evaluation and instrumental gas chromatographic analysis of edible oils".- J. Food Sci. 46, 1453 1456.

Min, D. B. y Wen, J. (1983a).- "Effects of dissolved free oxygen on the volatile compounds of oils".- J. Food Sci. 48, 1429-1430
Min, D. B. (1983b).- "Analyses of flavor qualities of vegetable oils by gas chromatography".- J. Am. Oil Chemists' Soc. 60, 544-545.

Montaño, A.; Sánchez, A. H. y Rejano, L. (1990).- "Rapid quantitative analysis of headspace components of green olive brine".- $\mathrm{J}$. Chromatogr. 521, 153-157.

Mordret, F.; Morin, O. y Coustille, J. L. (1985).- "Dètermination des flaveurs de corps gras".- Rev. Fr. Corps Gras 32, 193-200.

Moret, I.; Scarponi, G. y Cescon, P. (1984).- "Aroma components as discriminating parameters in the chemometric classification of venetian white wines".- J. Sci. Food Agric. 35, 1004-1011.

Morrison III, W. H.; Lyon, B. G. y Robertson, J. A. (1981).- "Correlation of gas liquid chromatographic volatiles with flavor intensity scores of stored sunflower oils".- J. Am. Oil Chemists' Soc. 58, 23-27.

Mottram, D. S.; Edwards, R. A. y MacFie, H. J. H. (1982).- "A comparison of the flavour volatiles from cooked beef and pork meat systems".- J. Sci. Food Agric. 33, 934-944.

Murray, K. E. (1977).- "Concentration of headspace, airborne and aqueous volatiles on Chromosorb 105 for examination by gas chromatography and gas chromatography-mass spectrometry".- J. Chromatogr. 135, 49-60.

Narain, N.; Hsieh, T. C. Y. y Johnson, C. H. (1990).- "Dynamic headspace concentration and gas chromatography of volatile flavor components in peach".- J. Food Sci. 55, 1303-1307.

Nawar, W. W.; Yoo, Y. J.; Bradley, M. S.; Morin, O.; Potter, T. y Whiteman R. C. (1988).- "A study of the volatile components generated from butteroil by heat".- Rev. Fr. Corps Gras 35, 117-122.

Nishimura, O.; Yamaguchi, K.; Mihara, S. y Shibamoto, T. (1989).- "Volatile constituents of guava fruits (Psidium guajava, L.) and canned puree". J. Agric. Food Chem. 37, 139-142.

Núñez, A. J.; González, L. F. y Janak, J. (1984).- "Preconcentration of headspace volatiles for trace organic analysis by gas chromatography".- J. Chromatogr. 300, 127-162.

Núñez, A. J.; Maarse, H. y Bemelmans, J. M. H. (1985).- "Volatile flavour components of grapefruit juice (citrus paradisi Macfadyen)".- J. Sci. Food Agric. 36, 757-763.

Olias, J. M.; Del Barrio, A. y Gutiérrez, R. (1977).- "Componentes volátiles en el aroma del aceite de oliva virgen. I.".- Grasas y Aceites 28, 107 112.

Olias, J. M.; Dobarganes, M. C.; Gutiérrez, F. y Gutiérrez, R. (1978)."Componentes volátiles en el aroma del aceite de oliva virgen. II. Identificación y análisis sensorial de los eluyentes cromatográficos". Grasas y Aceites 29, 211-218.

Olias, J. M.; Gutiérrez, F.; Dobarganes, M. C. y Gutiérrez, R. (1980). "Componentes volátiles en el aroma del aceite de oliva. IV. Su evolución e influencia en el aroma durante el proceso de maduración de los frutos en las variedades Picual y Hojiblanca".- Grasas y Aceites 31, 391-402.

Owuor, P. O.: Obaga, S. O. y Othieno, C. O. (1990).- "The effects of altitude on the chemical composition of black tea".- J. Sci. Food Agric. 50, 9-17

Paillard, N.: Pitoulis, S. y Mattei, A. (1970).- "Techniques de preparation et analyse de l'arome de quelques fruits".- Lebensm.- Wiss. Technol. 3, 107-114

Pino, J.; Rosado, A. y Baluja, R. (1988).- "Analysis of natural citrus aqueous essences". - Nahrung 32, 823-830.

Raghavan, S. K.; Reeder, S. K. y Khayat, A. (1989).- "Rapid analysis of vegetable oil flavor quality by dynamic headspace capillary gas chromatography".- J. Am. Oil Chemists' Soc. 66, 942-947.

Ramstad, T. y Nestrick, T. J. (1980).- "Purgue vessel design in determinations of volatile organic compounds".- Anal. Chim. Acta 121 345-348

Riba, M. L.: Tsiropoulos, N. y Torres, L. (1988).- "Efficiency of adsorptiondesorption of volatile compounds. Application to isoprene on Tenax GC".- J. Chromatogr. 437, 139-146

Rubico, S. M.; Resurrección, A. V. A. y Beuchat, L. R. (1988).- "Comparison of sensory properties and headspace volatiles of a peanut beverage processed at different temperature and time conditions".- J. Food Sci. $53,176-180$.

Rijks, J.; Curvers, J.; Noy, Th. y Cramers, C. (1983).- "Possibilities and limitations of steam distillation-extraction as a pre-concentration technique for trace analysis of organics by capillary gas chromatography".- J. Chromatogr. 279, 395-407.

Russell, L. F.; Quamme, H. A. y Gray, J. I. (1981).- "Qualitative aspects of pear flavor".- J. Food Sci. 46, 1152-1158.

Sakho, M.; Crouzet, J. y Seck, S. (1985).- "Volatile components of African Mango".- J. Food Sci. 50, 548-550.

Sanders, T. H. y Greene, R. L. (1989).- "The relationship of peanut maturity to 2-methylpropanal in headspace volatiles".- J. Am. Oil Chemists' Soc. $66,576-580$ 
Schultz, T. H.; Flath, R. A.; Mon, T. R.; Egglins, S. B. y Teranishi, B. (1977).- "Isolation of volatile compounds from a model system".- J. Agric. Food chem. 25, 446-449.

Selke, E. y Frankel, E. N. (1987).- "Dynamic headspace capillary gas chromatographic analysis of soybean oil volatiles".- J. Am. Oil Chemists' Soc. 64, 749-753.

Senf, L. y Frank, H. (1990).- "Thermal desorption of organic pollutants enriched on activated carbon. $V$. Desorption behaviour and temperature profile".- J. Chromatogr. 520, 131-135.

Shaw, P. E. y Wilson III, C. W. (1982).- "Volatile constituents of loquat (Eriobotrya japonica Lindl.) fruit".- J. Food Sci. 47, 1743-1744.

Shaw, G. J.; Ellingham, P. J. y Birch, E. J. (1983).- "Volatile constituents of feijoa-headspace analysis of intact fruit".- J. Sci. Food Agric. 34, 743-747.

Shaw, G. J.; Allen, J. M. y Yates, M. K. (1990).- "Volatile flavour constituents of feijoa (Feijoa sellowiana). Analysis of fruit flesh".- J. Sci. Food Agric. 50, 357-361

Shiota, H.; Young, H.: Paterson, V. J. y Irie, M. (1988).- "Volatile aroma constituents of Pepino fruit". - J. Sci. Food Agric. 43, 343-354.

Snyder, J. M.; Frankel, E. N. y Selke, E. (1985).- "Capillary gas chromatographic analyses of headspace volatiles from vegetable oils". J. Am. Oil Chemists' Soc. 62, 1675-1679.

Snyder, J. M.; Frankel, E. N.; Selke, E. y Warner, K. (1988).- "Comparison of gas chromatographic methods for volatile lipid oxidation compounds in soybean oil".- J. Am. Oil Chemists' Soc. 65, 1617-1620.

Snyder, J. M. y Mounts, T. L. (1990).- "Analysis of vegetable oil volatiles by multiple headspace extraction".- J. Am. Oil Chemists' Soc. 67 , 800-803.

Solinas, M.: Angerosa, F. y Cucurachi, A. (1985).- "Connessione tra prodotti di neoformazione ossidativa delle sostanze grasse e insorgenza del diffeto di ranciditá all'esame organolettico. Nota l".- Riv. Ital. Sci. Alim. 14, 361-368.

Solinas, M.; Angerosa, F. y Cucurachi, A. (1987a).- "Connessione tra prodotti di neoformazione ossidativa delle sostanze grasse e insorgenza del diffeto di ranciditá all'esame organolettico. Nota 2. Determinazione quantitativa".- Riv. Ital. Sostanze Grasse 64, 137145.

Solinas, M.; Marsilio, V. y Angerosa, F. (1987b).- "Evoluzione di alcuni componenti dell'aroma degli oli vergini di oliva in relazione al grado $\mathrm{d}$ maturazione delle olive".- Riv. Ital. Sostanze Grasse 64, 475-480.

Solinas, M.; Angerosa, F. y Marsilio, V. (1988a).- "Indagine su alcun componenti dell'aroma degli oli vergini di oliva in relazione alla varietà delle olive".- Riv. Ital. Sostanze Grasse 65, 361-368.

Solinas, M.; Angerosa, F. y Camera, L. (1988b).- "Evoluzione ossidativa di oli vegetali durante la frittura: determinazione dei componenti volatill mediante HRGC e HPLC".- Riv. Ital. Sostanze Grasse 65, 567-574.

Suzuki, J. y Bailey, M. E. (1985).- "Direct sampling capillary GLC analysis of flavor volatiles from ovine fat". - J. Agric. Food Chem. 33, 343-347.

Swinnerton, J. W.; Linnenbom, V. J. y Cheek, C. H. (1962a)."Determination of dissolved gases in aqueous solutions by gas chromatography".- Anal. Chem. 34, 483-485.
Swinnerton, J. W.; Linnenbom, V. J. y Cheek, C. H. (1962b).- "Revised sampling procedure for determination of dissolved gases in solution by gas chromatography".- Anal. Chem. 34, 1509.

Takeoka, G. R.; Flath, R. A.; Güntert, M. y Jennings, W. (1988).- "Nectarine volatiles: vacuum steam distillation versus headspace sampling".- $\mathrm{J}$. Agric. Food Chem. 36, 553-560.

Talou, T.; Delmas, M. y Gaset, A. (1989).- "Analysis of headspace volatiles from entire black truffle (Tuber malanosporum)".- J. Sci. Food Agric. 48, 57-62.

Ullich, F. y Grosch, W. (1988).- "Flavour deterioration of soya-bean oil: Identification of intese odour compounds formed during flavour reversion".- Fat Sci. Technol. 90, 332-336.

Urbach, G. (1987).- "Dynamic headspace gas chromatography of volatile compounds in milk".- J. Chromatogr. 404, 163-174.

Vejaphan, W.; Hsieh, T. C.-Y; Williams, S. S. (1988).- "Volatile flavor components from boiled crayfish (Procambanes clarkii) tail meat".- J. Food Sci. 53, 1666-1670.

Warner, K. y Frankel, E. N. (1985).- "Flavor stability of soybean oil based on induction periods for the formation of volatile compounds by gas chromatography".- J. Am. Oil Chemists' Soc. 62, 100-103.

Warner, K.; Frankel, E. N. y Moulton, K. J. (1988).- "Flavor evaluation of crude oil to predict the quality of soybean oil".- J. Am. Oil Chemists' Soc. 65, 386-391.

Werkhoff, P. y Bretschneider, W. (1987a).- "Dynamic headspace gas chromatography: concentration of volatile components after thermal desorption by intermediate cryofocusing in a cold trap. I. Principle and applications".- J. Chromatogr. 405, 87-98.

Werkhoff, P. y Bretschneider, W. (1987b).- "Dynamic headspace gas chromatography: concentration of volatile components after thermal desorption by intermediate cryofocusing in a cold trap. II. Effect of sampling and desorption parameters on recovery".- J. Chromatogr. 405, 99-106.

Weurman, c. (1969).- "Isolation and concentration of volatiles in food odor research".- J. Agric. Food Chem. 17, 370-384.

Wyatt, D. M. (1987).- "Dynamic headspace gas chromatography/mass spectrometry technique for determining volatiles in ambient stored vegetable oils".- J. Chromatogr. Sci. 25, 257-261.

Wyllie, B. G.; Brophy, J. J.; Sarafis, V. y Hobbs, M. (1990).- "Volatile components of the fruit of Pistacia lentiscus".- J. Food Sci. 55, 13251326.

Yasuhara, A. (1987).- "Comparison of volatile components between fresh and rotten mussels by gas chromatography-mass spectrometry".- $J$. Chromatogr. 409, 251-258.

Young, H.; Paterson, V. J. y Burns; D. J. W. (1983).- "Volatile aroma constituents of kiwifruit".- J. Sci. Food Agric. 34, 81-85.

Young, H. y Paterson, V. J. (1985).- "The effects of harvest maturity, ripeness and storage on kiwifruit aroma".- J. Sci. Food Agric. 36, 352358.

(Recibido: Octubre 1991). 